\title{
A NOVEL NONCONTACTING WAVEGUIDE BACKSHORT FOR SUBMILLIMETER WAVE FREQUENCIES
}

\author{
W. R. McGrath, ${ }^{1}$ T. M. Weller, ${ }^{2}$ and L. P. B. Katehi ${ }^{2}$ \\ ${ }^{I}$ Center for Space Microelectronics Technology, Jet Propulsion \\ Laboratory, California Institute of Technology, 4800 Oak Grove Drive \\ Pasadena, CA 91109
}

${ }^{2}$ Radiation Laboratory, University of Michigan, Ann Arbor, MI 48109

Received November 21, 1994

\begin{abstract}
$\underline{\text { Abstract }}$
A novel noncontacting waveguide backshort has been developed for millimeter wave and submillimeter wave frequencies. It employs a metallic bar with rectangular or circular holes. The size and spacing of the holes are adjusted to provide a periodic variation of the guide impedance on the correct length scale to give a large reflection of rf power. This design is mechanically rugged and can be readily fabricated for high submillimeter wave frequencies where conventional backshorts are difficult or impossible to fabricate. Model experiments have been performed at $4 \mathrm{GHz}-6 \mathrm{GHz}$ to empirically optimize the design parameters. Values of reflected power greater than $95 \%$ over a $30 \%$ bandwidth have been achieved. A specific design is presented which has also been successfully scaled to WR-10 band $(75 \mathrm{GHz}-110 \mathrm{GHz})$. A theoretical analysis is compared to the experiments and found to agree well with the measured data.
\end{abstract}

Key words: submillimeter wave, waveguide, backshort, tuning element

\section{Introduction}

Rectangular waveguides are used in a wide variety of applications and instruments covering frequencies from low microwave to high submillimeter wave bands. A frequent use of waveguide is as a variable length transmission line. Such a line serves as an adjustable rf tuning element in complex circuits and is formed by placing a movable shortcircuit or "backshort" in the waveguide. A common example is a contacting backshort where a springy metal, such as beryllium copper, makes DC contact with the waveguide broadwalls. These backshorts are 
generally excellent and essentially frequency independent. However, they are susceptable to wear from sliding friction and are difficult to fabricate for frequencies above a few hundred $\mathrm{GHz}$ where waveguide dimensions are small; for WR-3 band $(220 \mathrm{GHz}-325 \mathrm{GHz})$ for example, the dimensions are $0.864 \mathrm{~mm} x 0.432 \mathrm{~mm}$.

Another approach, commonly used at millimeter wave frequenices, is the noncontacting backshort [1,2,3]; an example is shown in Fig. 1. A thin insulator, such as mylar [4], prevents DC contact. In order to obtain a large reflection of if power, a noncontacting backshort provides a series of properly phased reflections, which result from cascaded high-impedance and low-impedance transmission line sections. These sections are approximately $\lambda_{\mathrm{g}} / 4$ long where $\lambda_{\mathrm{g}}$ is the guide wavelength. The $\mathrm{rf}$ impedance at the reference plane at the front of this backshort is given approximately by

$$
Z_{r f}=\left(\frac{Z_{l o w}}{Z_{\text {high }}}\right)^{n} \cdot Z_{\text {low }}
$$

where $Z_{\text {low }}$ is the guide impedance of the thick (low-impedance) section and $Z_{\text {high }}$ is the impedance of the thin (high-impedance) section and $n$ is the number of sections. While good performance is possible [1] (i.e., $Z_{\text {rf }}$ $\ll Z_{g}$, where $Z_{g}$ is the characteristic waveguide impedance), this design is not readily scalable to submillimeter wave frequencies (above $300 \mathrm{GHz}$ ). The high-impedance sections would become too thin and weak to allow the backshort to slide snugly inside the waveguide. In principle, a single thick bar would give a large reflection due to the large impedance

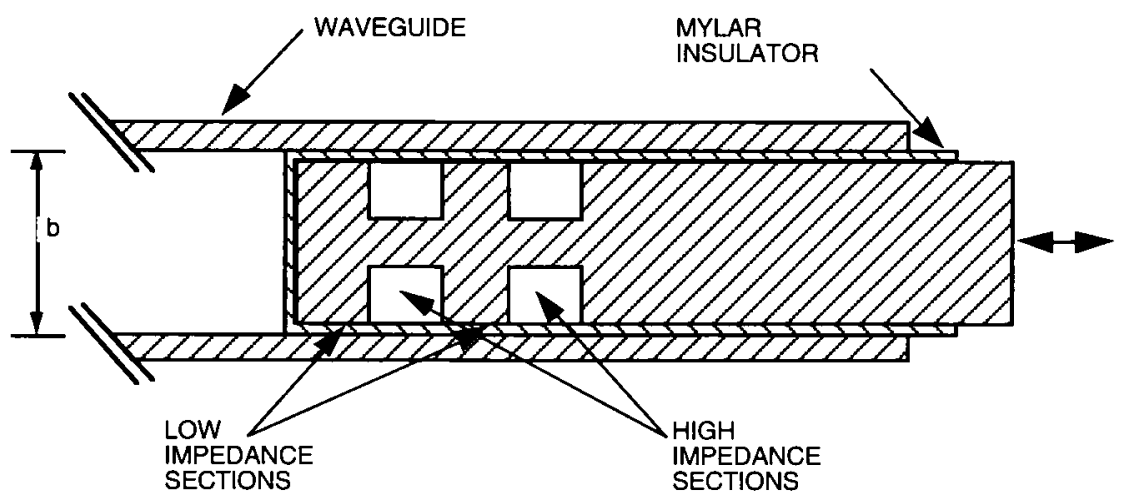

Figure 1. Cross sectional view of a conventional noncontacting backshort. "b" is the waveguide height. 
discontinuity, but only over a narrow bandwidth. Deep dropouts (bands of low reflection) would be present and would depend on the overall length of the bar inserted into the waveguide (this is discussed in section V.A.).

\section{Novel Noncontacting Backshort Design}

A novel noncontacting waveguide backshort has been developed [5,6], and is shown in Fig. 2, which addresses the problems associated with conventional backshorts at high frequencies. The appropriate reflections are achieved by either rectangular or circular holes, with the proper dimension and spacings, cut into a metallic bar. The bar is fabricated to form a close fit inside the waveguide with a mylar insulator and small air gap along the broadwalls. The holes replace the thin, highimpedance sections in the conventional design (see Fig. 1). Since the holes extend completely through the bar, this yields a higher impedance than in the conventional design. Thus, the high-to-low impedance ratio is larger which improves the bandwidth. In addition, the electromagnetic fields of the dominant $\mathrm{TE}_{10}$ mode are concentrated near the central axis of the waveguide, thus making the holes effective in producing a large overall reflection of if power.

This backshort is readily fabricated using a variety of techniques. For submillimeter wave frequencies, above $300 \mathrm{GHz}$, the metallic bar is a piece of shim stock polished to the correct thickness. The holes can be formed by drilling, punching, laser machining, or they can be etched using lithography techniques. Silicon micromachining techniques [7] can be used when more precise tolerances are required. Thin oxide layers can be deposited to serve as the insulator [3].

In this paper, the experimental models and $\mathrm{rf}$ measurements at $4 \mathrm{GHz}-6 \mathrm{GHz}$ used to empirically optimize the backshort reflection coefficient and bandwidth will be described. Specific optimized designs are presented here which can be scaled to any desired frequency. In particular, results are presented for a backshort scaled to W-band $(75 \mathrm{GHz}$ - $110 \mathrm{GHz}$ ) to demonstrate the performance at high millimeter wave frequencies.

\section{Theoretical Formulation}

Since low frequency (i.e., large-scale) modeling methods can be time consuming, a theoretical formulation was developed which could be used to analyze and predict the performance of the backshort. This theory employs a hybrid approach that combines the mode-matching method with an integral equation technique. In the first part of the analysis, the scattering matrix at the reference plane of the backshort is calculated (see 
(a)

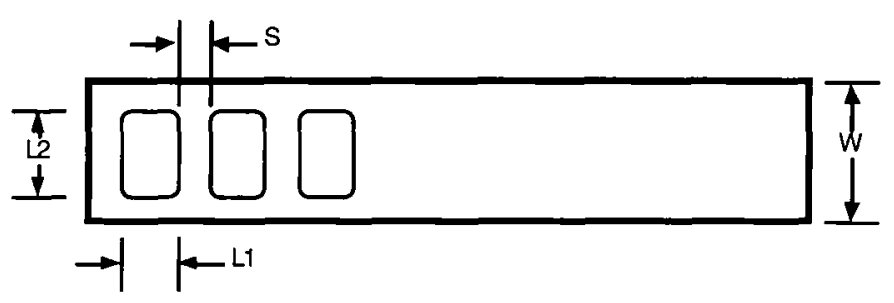

(b)
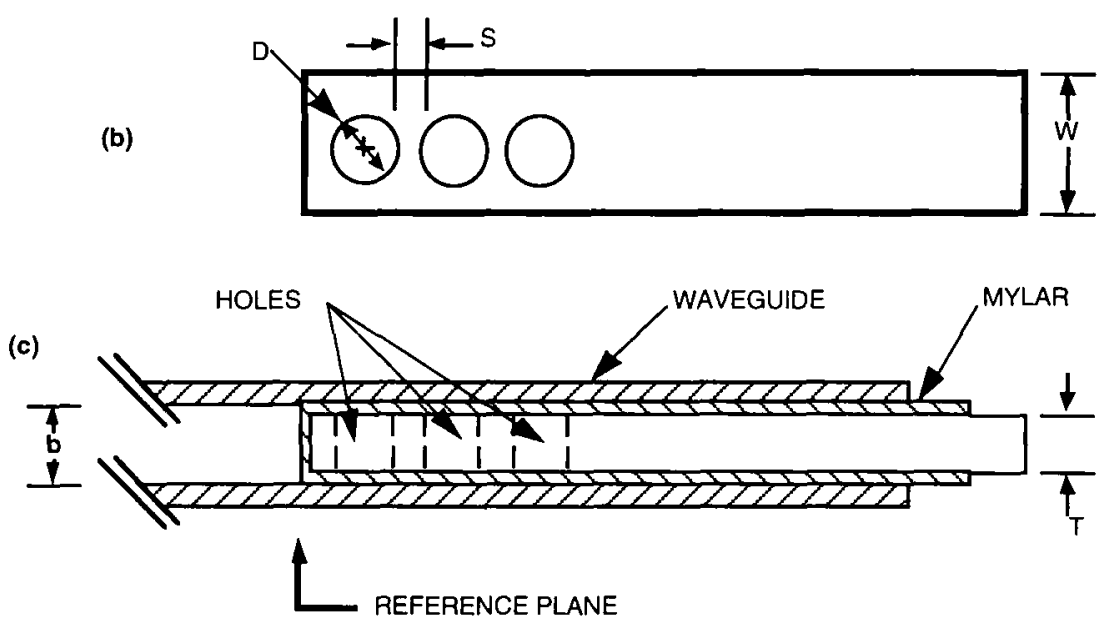

Figure 2. New noncontacting backshort design. (a) A metallic bar of width $W$ and thickness $T$ with rectangular holes cut near one end. The hole length $\mathrm{L} 1$ and separation $S$ are important in determining the rf properties. " $S$ " is also the distance from the end of the bar to the edge of the first hole. (b) A similar backshort design using circular holes. " $\mathrm{D}$ " is the hole diameter. (c) Cross sectional view of the new backshort in the waveguide. A thin mylar insulator allows the backshort to slide smoothly.

Fig. 2(c)). Then, given the dominant waveguide mode incident on the backshort, the resultant fields in the dielectric (mylar) filled gap along the broadside of the backshort can be determined. The next step is to solve for the reflection of these scattered modes due to the presence of the holes. This is done by assuming fictitious metal surfaces cover the holes, upon which equivalent magnetic currents are imposed. An integral equation can then be derived which involves the unknown currents and the fields from the incident dominant mode, by enforcing field continuity requirements across the hole apertures. After solving the integral equation using the method of moments, the initial scattering at the front of the backshort is combined with the additional radiation from the magnetic currents to obtain the total reflection of the dominant waveguide mode. 
In order to compare the theory to the measurements, the dielectric constant $\varepsilon_{\mathrm{r}}$ of the insulator surrounding the backshort must be known, and the load impedance $Z_{\ell}$ at the point where the backshort exits the waveguide is required. As shown below, the reflection coefficient and bandwidth are not strongly dependent on $\mathrm{Z}_{\ell}$. The insulator, however, has a significant influence. Since the insulator, typically mylar, does not completely or even uniformly fill the gap between the backshort and the waveguide wall, an effective dielectric constant $\varepsilon_{\text {reff }}$ must be used. The best value for $\varepsilon_{\text {reff }}$ is obtained by fitting the theory to the experimentally measured response. However for design purposes, a close approximation

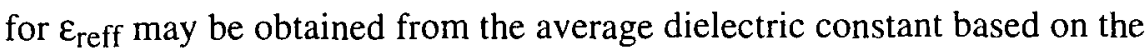
fraction of the gap filled by the mylar:

$$
\varepsilon_{r e f f}=\frac{t_{d}}{y_{g}} \cdot\left(\varepsilon_{r}-1\right)+1
$$

where $t_{d}$ is the thickness of the dielectric and $y_{g}$ is the overall height of the gap between the backshort and the waveguide wall. The guide wavelength can then be calculated using [8]:

$$
\lambda_{g}=\frac{\lambda}{\sqrt{\varepsilon_{\text {reff }}-\left(\lambda / \lambda_{c}\right)^{2}}}
$$

where $\lambda$ is the free space wavelength and $\lambda_{c}$ is the cutoff wavelength of the air-filled waveguide. In the limit that the mylar lies perfectly flat against the backshort, the transverse mode technique [9] can be used to give an accurate value for the propagation constant and hence the guide wavelength in the dielectric filled gap.

A complete discussion of the theoretical analysis will be presented elsewhere [10]. In this paper, the analysis is compared with the experimentally measured performance. Good agreement is found which establishes the validity of the theory.

\section{Measurement Techniques}

The backshort design was optimized by testing the performance in WR-187 band waveguide $(3.16 \mathrm{GHz}-6.32 \mathrm{GHz})$. This waveguide band was chosen because if measurements are convenient in this frequency range and the large waveguide dimensions, $47.8 \mathrm{~mm} x 22.2 \mathrm{~mm}$, simplify 
construction of the backshorts. Several backshorts were machined out of aluminum and tested. The backshort dimensions were typically $\mathrm{W}=$ $47.5 \mathrm{~mm}$ and $\mathrm{T}=19.7 \mathrm{~mm}$, leaving a gap of $1.25 \mathrm{~mm}$ between either side of the bar and the waveguide broad wall. This is a large gap, but it corresponds to typical fabrication tolerances to be expected for much smaller waveguides at $200 \mathrm{GHz}-300 \mathrm{GHz}$. Thus, the results should correspond closely to those expected for actual high-frequency backshorts in practical applications. The parameters of the backshort which were varied are the (a) shape of the holes, (b) size and spacing of the holes, (c) number of holes, and (d) the thickness of the mylar insulator. Each of these parameters can affect the electrical length of the high- and lowimpedance sections which, in turn, determines the rf performance of the backshort.

The magnitude and phase of the reflection coefficient were measured from $4 \mathrm{GHz}$ to $6 \mathrm{GHz}$ with an $\mathrm{HP} 8510 \mathrm{~B}$ Vector Network Analyzer. A commercially available coaxial-to-waveguide transition was used to connect the waveguide to the network analyzer. This measurement system was calibrated using: 1) two offset contacting shorts set at $\lambda_{\mathrm{g}} / 8$ and $3 \lambda_{\mathrm{g}} / 8$ from the reference plane in the waveguide and 2) a wedge-shaped sliding waveguide load. Subsequent verification using a contacting short indicated a measurement error of about $\pm 0.15 \mathrm{~dB}$ in the magnitude. In addition, a few measurements were made of the reflection coefficient magnitude using a Wiltron Scalar Network Analyzer and an SWR bridge.

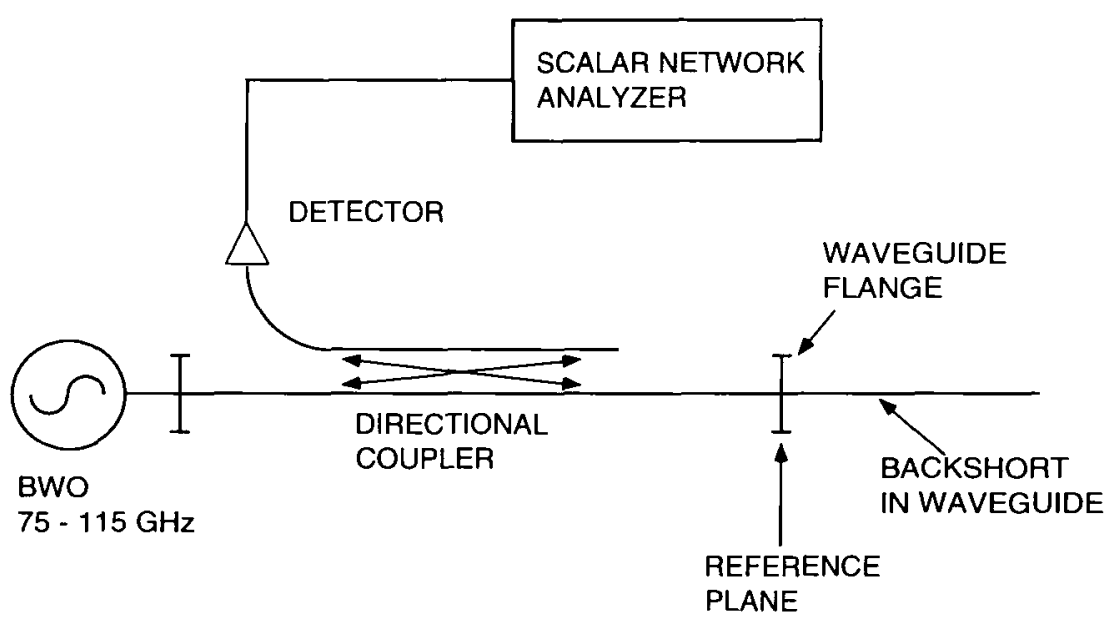

Figure 3. Millimeter wave test apparatus. A copper plate at the waveguide flange is used to provide $0 \mathrm{~dB}$ reference level. 
Millimeter wave tests were made on backshorts scaled for use in WR-10 waveguide at $75 \mathrm{GHz}-110 \mathrm{GHz}$. A block diagram of the test system is shown in Fig. 3. A Micro-Now backward wave oscillator (BWO) and a Singer sweeper were used to provide a $75 \mathrm{GHz}-110 \mathrm{GHz}$ swept signal. A direct detector, $10 \mathrm{~dB}$ directional coupler, and Wiltron 560A Scalar Network Analyzer were used to detect the reflected power. The system was calibrated by placing a copper plate at the position of the reference plane at the waveguide flange to provide a $0 \mathrm{~dB}$ reference level.

\section{WR-187 Band Measurements: Results and Comparison With Theory}

\section{V-A: Backshort with No Holes}

Figure 4 shows the reflected signal for a solid bar without holes. The mylar insulator is $0.89 \mathrm{~mm}$ thick and hence fills about $75 \%$ of the gap between the waveguide broad wall and the backshort (we use plain mylar sheets, without any adhesive which could add to the overall dielectric thickness). As seen in Fig. 4, a solid bar does not make a good backshort. Deep dropouts (reflection much less than $-1.0 \mathrm{~dB}$ ) are observed at several frequencies. Figure 5 shows that the theoretical analysis also indicates deep dropouts for this case. As discussed above and elsewhere [10], the effective dielectric constant $\varepsilon_{\text {reff }}$ and the load impedance $Z_{\ell}$ at the point

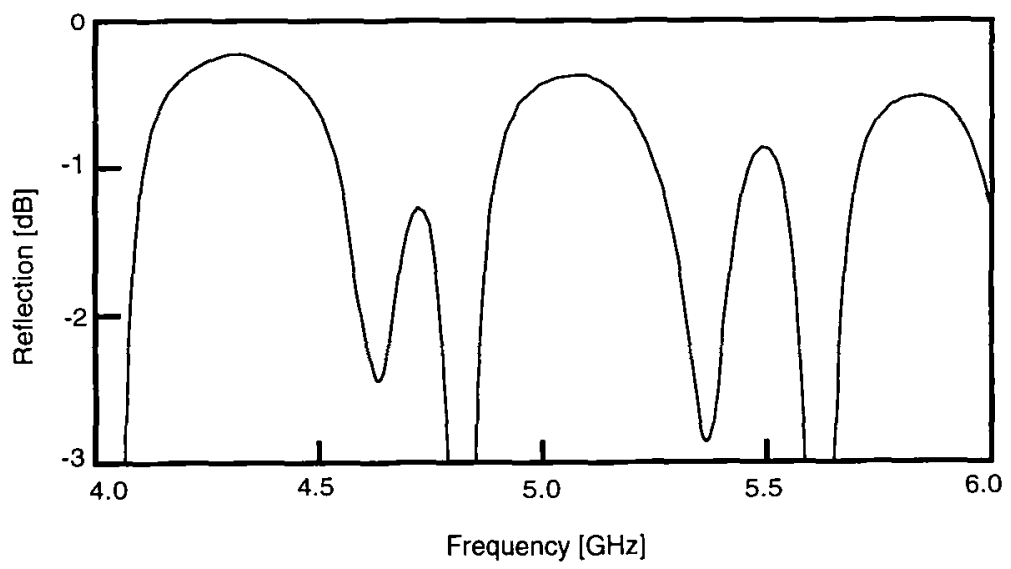

Figure 4. Reflected power from a solid bar without holes. This design does not make a good backshort. Several large dropouts occur across the frequency band. 


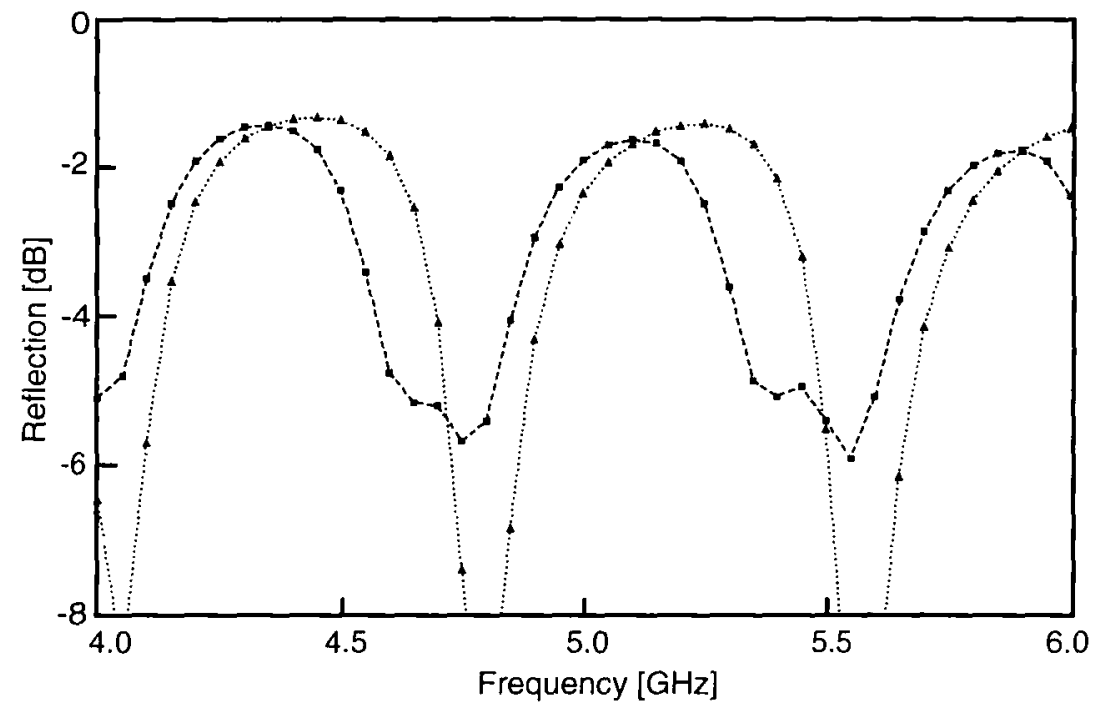

Figure 5. Calculated reflected power versus frequency for a solid bar without holes using the theoretical formulation [10]. The dotted line was calculated assuming the bar was symmetrically placed in the waveguide with the same effective dielectric constant in the gap on each side. Offsetting the bar slightly in the waveguide creates different gaps on each side with slightly different effective dielectric constants, giving rise to the double-dip dropouts shown by the dashed line.

where the backshort exits the waveguide are required for the calculation. The dotted line in Fig. 5 was calculated assuming the bar was symmetrically placed in the waveguide. The same value of effective dielectric constant, $\varepsilon_{\text {reff }}=2.4$, was used for the gap on each side and the normalized load impedance was chosen to be $Z_{\ell}=2+1 j$. Large single-dip dropouts are predicted, though double-dip dropouts are observed experimentally. The theory suggests these result from the bar not being exactly symmetrically positioned in the waveguide which would give different size gaps on each side of the bar with slightly different $\varepsilon_{\text {reff. }}$ This would lead to different resonant frequencies for the cavities formed in each of the gaps. This is another reason why a solid bar is not a good backshort since the resonant frequency of these cavities depend on how far the backshort is inserted into the waveguide. The dashed line in Fig. 5 was calculated using different effective dielectric constants in the gaps: $\varepsilon_{\text {reff } 1}=2.4$ and $\varepsilon_{\text {reff } 2}=2.6$. As can be seen, the double-dip structure is beginning to emerge. Properly designed holes in the bar will eliminate these dropouts. 


\section{V-B: Backshort with Rectangular Holes}

Figure 6(a-d) shows the performance for an optimized backshort with three rectangular holes for mylar thicknesses of $0.64 \mathrm{~mm}, 0.76 \mathrm{~mm}$, $0.89 \mathrm{~mm}$, and $1.02 \mathrm{~mm}$, respectively (the thicknesses used for the various tests were obtained by stacking 2 to 5 layers of $0.127 \mathrm{~mm}$ and $0.254 \mathrm{~mm}$ thick non-adhesive mylar sheets). The three holes each have dimensions $\mathrm{L}_{1}=19.3 \mathrm{~mm}$ and $\mathrm{L}_{2}=28.4 \mathrm{~mm}$ with a spacing $\mathrm{S}=8.7 \mathrm{~mm}$ (see Fig. 2(a)). In Fig. 6(c), the reflection coefficient is greater than $-0.2 \mathrm{~dB}(95 \%$ reflected power) over a $33 \%$ bandwidth centered around $4.8 \mathrm{GHz}$. This is, of course, a dramatic improvement over the solid bar without holes and demonstrates the success of this new design. This is the best overall design tested and can be scaled for higher frequencies (see section VI for a millimeter wave test of this design). In addition, in Fig. 6(a), the response is slightly flatter overall than the other cases shown (the deep dropout has moved out of band for a mylar thickness of $0.64 \mathrm{~mm}$ ) except for a slight decrease in the reflection near $4.8 \mathrm{GHz}$. This response is similar to that obtained for a conventional noncontacting backshort

As can be seen in Fig. 6, the upper end of the useful band is limited by a deep dropout. This dropout decreases in frequency as the mylar thickness increases as a result of an increase in the effective dielectric constant $\varepsilon_{\text {reff }}$ in the gap between the bar and the waveguide broadwall. The dropout is caused by phased reflections at the front and back of the hole and is, thus, directly related to the length of the hole. As indicated by the theoretical analysis [10], the electrical length of the holes and spaces should lie between $65^{\circ}$ and $105^{\circ}$ for the best broadband performance. This range of electrical lengths is determined using an effective dielectric constant which is the same for both the low impedance and highimpedance (the region over the hole) sections. The same dielectric constant is used for the "air filled" hole since any power which leaks past the hole most likely does so in the narrow dielectric-filled gaps along the edges of the hole [10].

In Fig. 6(c), while the reflected power is flat over a broad frequency range, dropouts appear at the high end since the hole lengths are $\mathrm{L}_{1}=193^{\circ}$ at $5.75 \mathrm{GHz}$, which is very near $\lambda_{\mathrm{g}} / 2$. The effective dielectric constant used in this case is $\varepsilon_{\text {reff }}=2.4$. In contrast, the holes in Fig. 6(a) are less than half a wavelength long and, thus, no dropouts are seen. The exact shape of the dropout is affected by sideways displacements (perpendicular to the waveguide axis) allowed by the tolerance gap. However, the high reflection band is insensitive to such displacements. Thus, the backshort should work well at high frequencies (above $100 \mathrm{GHz}$ ) where such alignment errors are typical. 


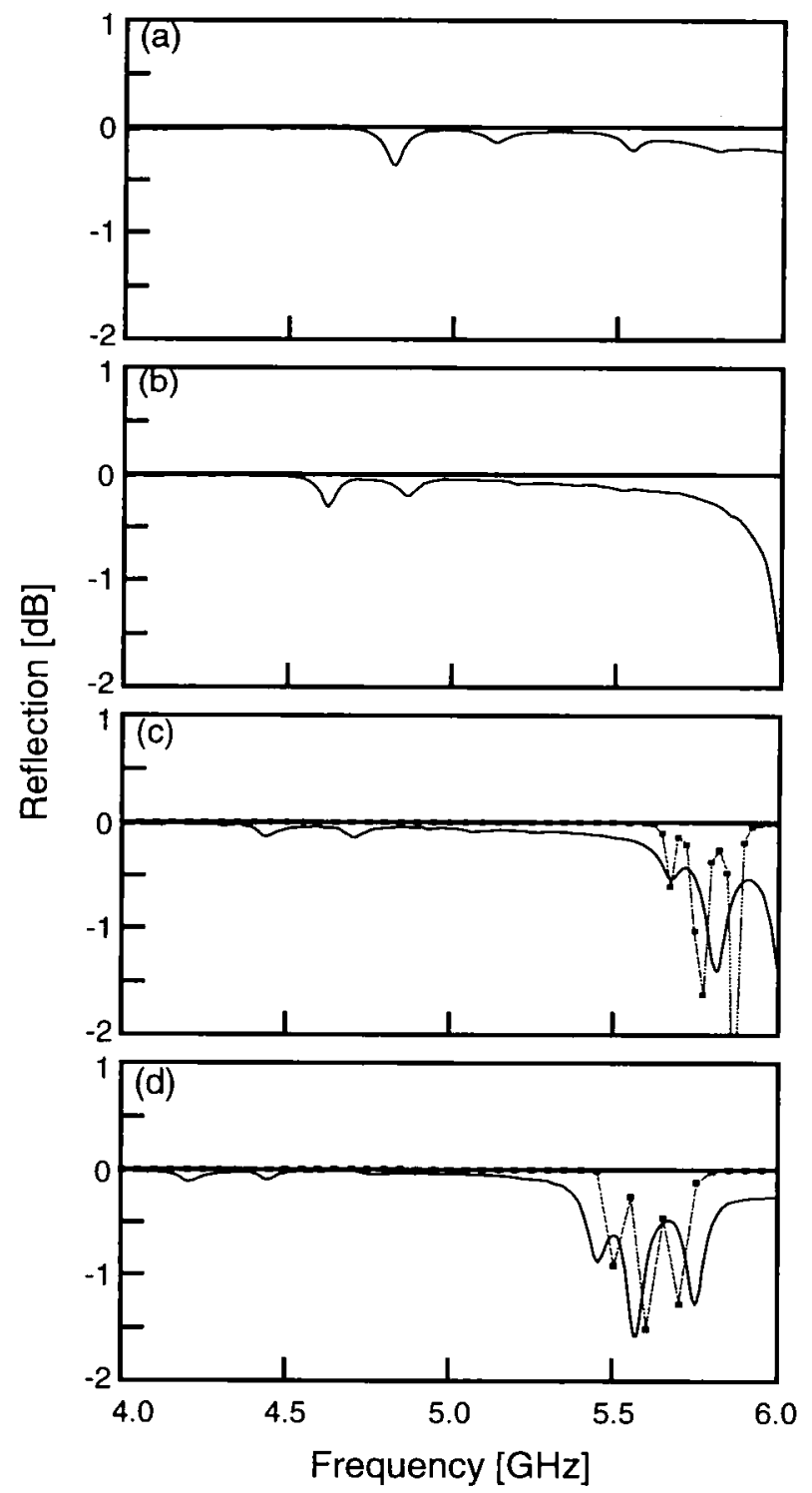

Figure 6. Reflected power from a backshort with three rectangular holes for mylar thicknesses of (a) $0.64 \mathrm{~mm}$, (b) $0.76 \mathrm{~mm}$, (c) $0.89 \mathrm{~mm}$, (d) $1.02 \mathrm{~mm}$. Excellent performance is obtained over a broad bandwidth. The calculated performance is shown in (c) and (d) (squares connected by dotted lines). 
The calculated response is also shown in Figures 6(c) and 6(d). As can be seen, the theoretical analysis correctly predicts the high reflection band and the deep dropout at the upper end of the band. The parameters used in this case are $\varepsilon_{\text {reff }}=2.4$ and $\mathrm{Z}_{\ell}=2+1 \mathrm{j}$ for Fig. $6(\mathrm{c})$ and $\varepsilon_{\text {reff }}=2.55$ and $Z_{\ell}=1.5+0 j$ for Fig. $6(\mathrm{~d})$. Changing $Z_{\ell}=2+1 j$ for Fig. 6(d) increased the depth of the calculated dropout but did not change its position. The high reflection band was essentially unaffected by this change. In general, the choice for $Z_{\ell}$ is not critical for frequency bands where the reflection is high since little power makes it to the point where the backshort exits the waveguide. The much smaller dip observed between $4 \mathrm{GHz}$ and $5 \mathrm{GHz}$ is not predicted by the theory. Its exact nature is not yet well understood but is possibly related to the excitation of TEM and/or higher order TE and TM modes which result from the asymmetric placement of the backshort in the waveguide [2]. As the mylar thickness increases, this dip diminishes since the thicker mylar fills the gaps on each side of the backshort more completely and hence tends to make the gaps equal in size. Nevertheless, this dip is small enough that it does not limit the performance of the backshort.

Figure 7 shows the measured phase versus frequency corresponding to Fig. 6(c). As can be seen, the phase varies smoothly with frequency near $180^{\circ}$ for the high reflection part of the band. This indicates that the backshort is behaving as a well defined short circuit. Small wiggles in the phase corresponding to the deep dropouts occur as expected near the upper end of the band. The calculated phase is also shown in Fig. 7 and the agreement is extremely good. The theory and experiment are almost indistinguishable, with only a small deviation at the upper end of the band near the dropouts.

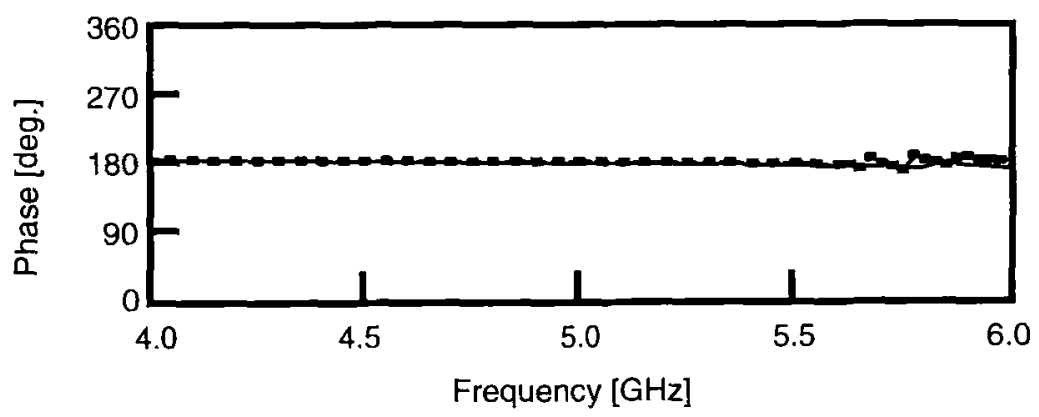

Figure 7. Measured (solid line) and calculated (squares) phase versus frequency for a backshort with three rectangular holes and a mylar thickness of $0.89 \mathrm{~mm}$ (this corresponds to the magnitude versus frequency plot in Fig. 6(c)). The phase varies smoothly around $180^{\circ}$ indicating a well-defined short circuit. 


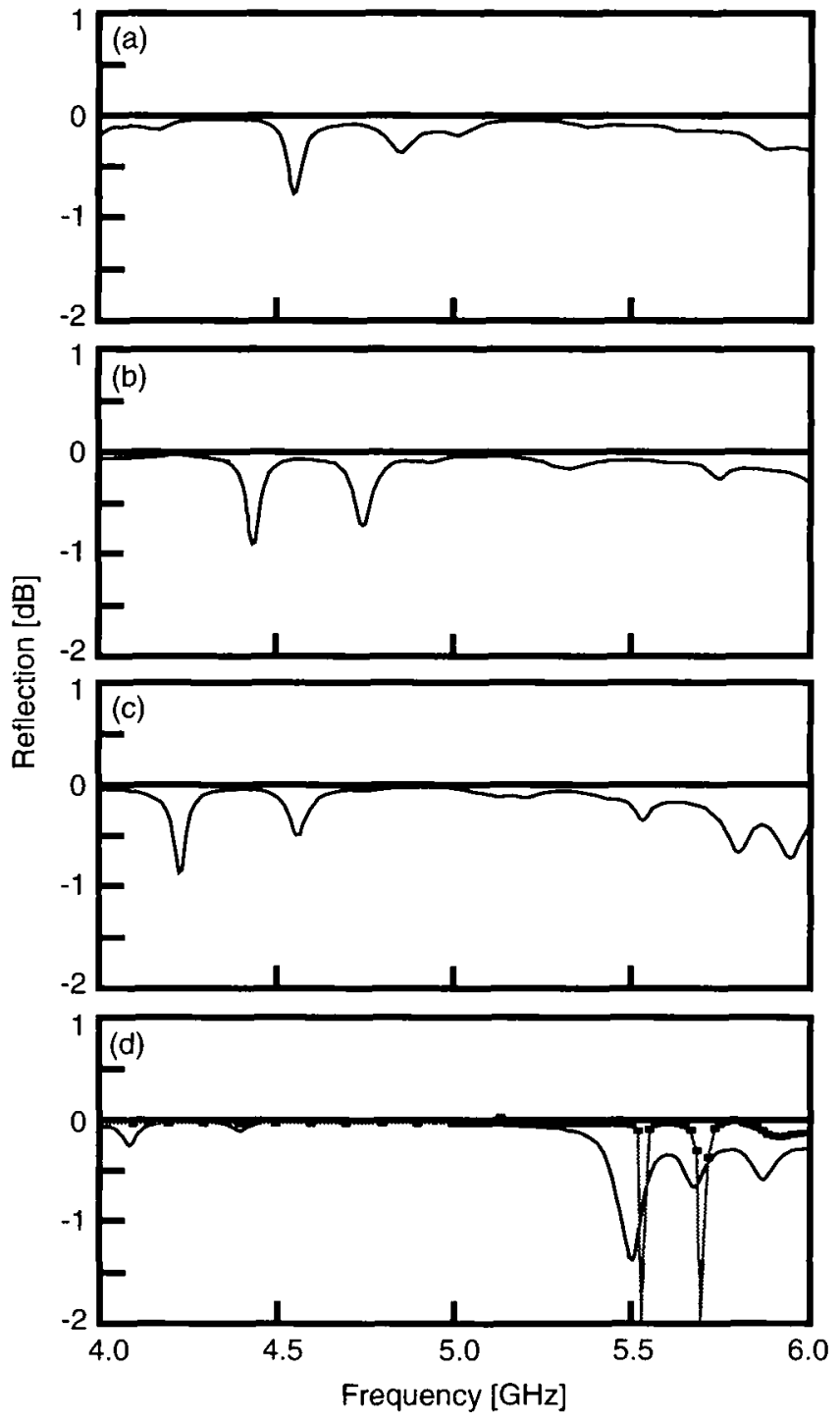

Figure 8. This figure shows the effect of reducing the rectangular hole dimensions $\mathrm{L}_{1}$ and $\mathrm{S}$ by $20 \%$ as compared with the case shown in Fig. 6 . The mylar thicknesses are the same as in Fig. 6: (a) $0.64 \mathrm{~mm}$, (b) $0.76 \mathrm{~mm}$, (c) $0.89 \mathrm{~mm}$, (d) $1.02 \mathrm{~mm}$. The performance is similar except that the small dips between $4 \mathrm{GHz}$ and $5 \mathrm{GHz}$ occur about $5 \%$ lower in frequence than the corresponding case in Fig. 6 . The calculated performance is shown in (d) (squares connected by gray line). 
Figure 8 shows the effect of reducing the hole dimensions $\mathrm{L}_{1}$ and $\mathrm{S}$ by $20 \%$ to $\mathrm{L}_{1}=15.5 \mathrm{~mm}$ and $\mathrm{S}=6.8 \mathrm{~mm}$. Four mylar thicknesses, 0.64 $\mathrm{mm}, 0.76 \mathrm{~mm}, 0.89 \mathrm{~mm}$ and $1.02 \mathrm{~mm}$, are shown in Fig. 8(a) - 8(d) respectively. These are compared to the corresponding cases in Fig. 6(a) 6(d). As can be seen, the performance is similar except that the two small dips between $4 \mathrm{GHz}$ and $5 \mathrm{GHz}$ are somewhat deeper. These dips move down in frequency as the mylar gets thicker as expected. However, we see that these dips occur about 5\% lower in frequency than the corresponding case in Fig. 6. This suggests that they are not directly related to the hole dimensions since reducing these parameters should have moved the dips $u p$ in frequency. Decreasing $\mathrm{L}_{1}$ and $\mathrm{S}$, however, increases the length of the "cavity" between the end of the last hole and the point where the backshort exits the waveguide. In fact, this "cavity" length increases by about $8 \%$. This would cause a corresponding decrease in any resonances associated with this cavity and supports the idea that these dips are related to power which leaks past the holes and interacts with this region of the backshort. As mentioned before, the theory does not account for any asymmetries in the sizes of these cavities and the resultant modes which may exist in them.

The calculated performance is shown in Fig. 8(d). The agreement is good at frequencies where the reflection is high. The theory also indicates the frequency range where the dropouts occur though the exact shape of the calculated dropouts differs from the measurements. Predicting this shape is of course less important than predicting the useful frequency range of the high reflection band.

\section{V-C: Backshort with Circular Holes}

Figure $9(\mathrm{a}-\mathrm{e})$ shows the performance for an optimized backshort with three circular holes for mylar thicknesses of $0.51 \mathrm{~mm}, 0.64 \mathrm{~mm}, 0.76$ $\mathrm{mm}, 0.89 \mathrm{~mm}$, and $1.02 \mathrm{~mm}$, respectively. The three holes each have diameter $\mathrm{D}=19.4 \mathrm{~mm}$ with a spacing $\mathrm{S}=8.6 \mathrm{~mm}$ (see Fig. 2(b)). In Fig. 9(d), for example, the reflection coefficient is greater than $-0.2 \mathrm{~dB}$ over a $32 \%$ bandwidth with a center frequency near $4.75 \mathrm{GHz}$. These results are similar to those obtained with the rectangular holes which is encouraging since round holes are easier to fabricate. This is the best design tested with round holes and can be readily scaled to higher frequencies (see section VI for a millimeter wave test). The bandwidth is limited, however, by dropouts at both the upper and lower end of the frequency band while the backshorts with rectangular holes showed no deep dropouts down to the cutoff frequency of the waveguide. As the mylar thickness is increased, the dropout at the lower end moves down out of the measurement range and another dropout appears at the upper end. The downward shift is expected with increasing dielectric thickness as previously discussed. 

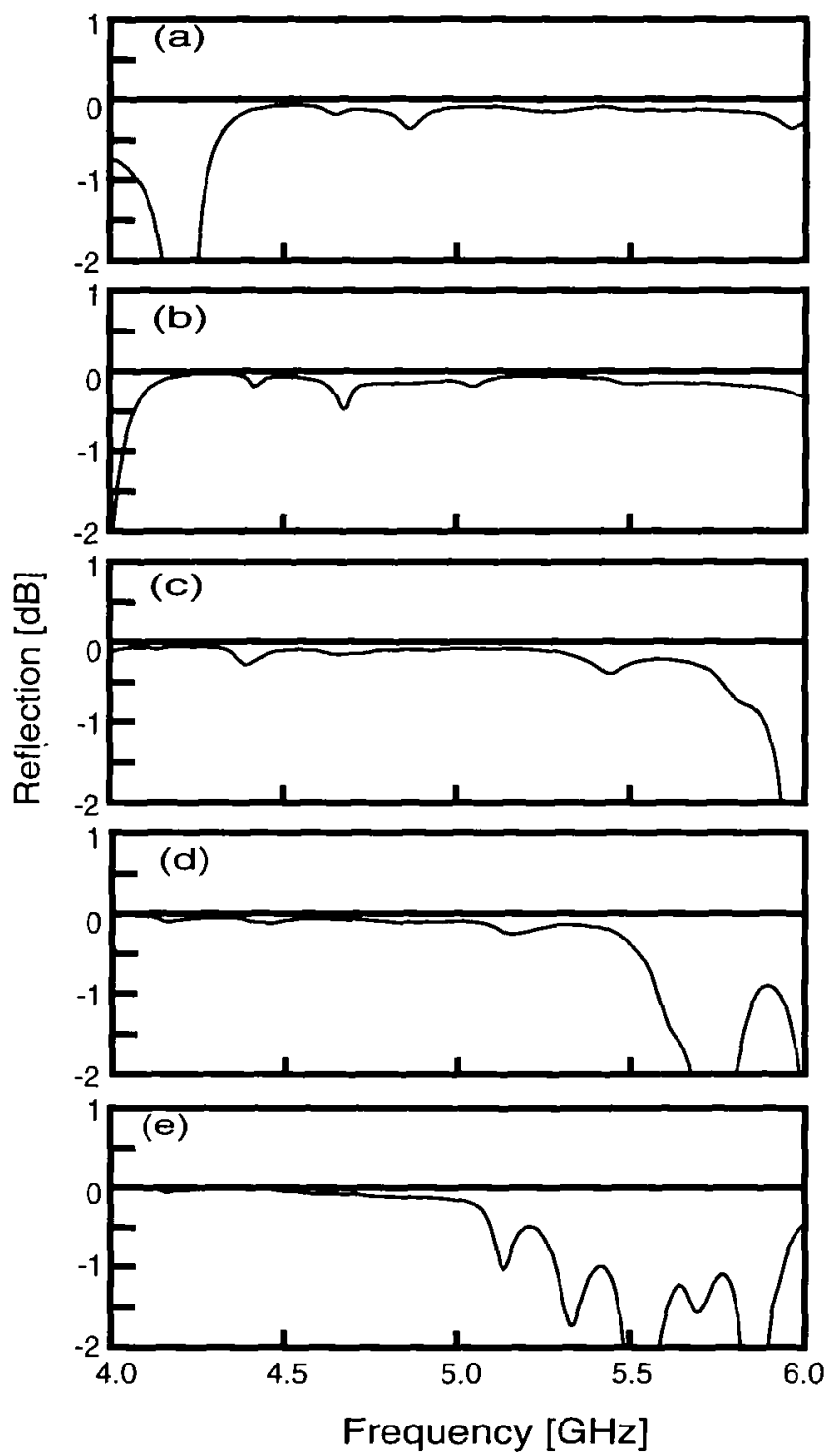

Figure 9. Reflected power from a backshort with three circular holes for mylar thicknesses of (a) $0.51 \mathrm{~mm}$, (b) $0.64 \mathrm{~mm}$, (c) $0.76 \mathrm{~mm}$, (d) $0.89 \mathrm{~mm}$, (e) $1.02 \mathrm{~mm}$. Excellent performance is obtained over a broad bandwidth similar to the backshort with rectangular holes. 


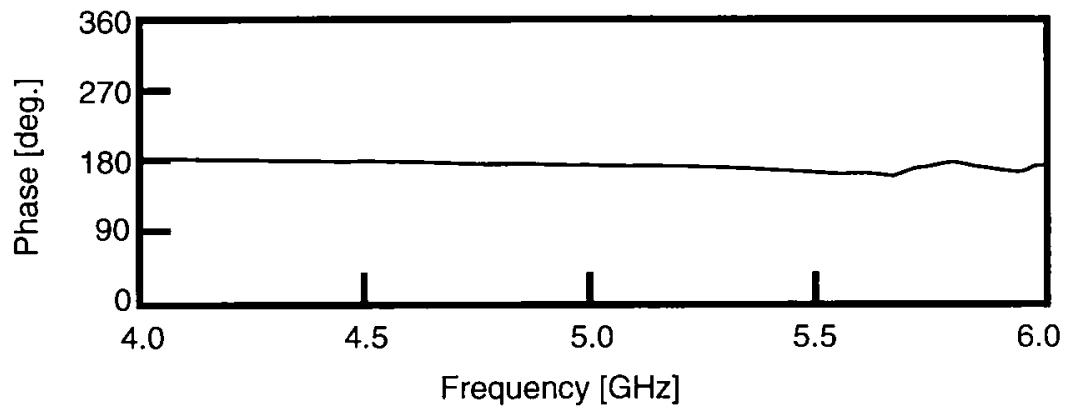

Figure 10. Measured phase versus frequency for a backshort with three circular holes and a mylar thickness of $0.89 \mathrm{~mm}$ (this corresponds to the magnitude versus frequency plot in Fig. 9(d)). The phase varies smoothly around $180^{\circ}$ indicating a well defined short circuit.

Figure 10 shows the measured phase versus frequency corresponding to Fig. 9(d) and, as with the rectangular hole design, indicates that the backshort is behaving as a well defined short circuit. Small wiggles in the phase occur only at frequencies corresponding to the deep dropouts as expected.

Figure 11 shows the effect of varying the hole diameter and spacing. In Fig. 11(a) both the diameter and spacing are decreased by a factor of 0.8 to $\mathrm{D}=15.4 \mathrm{~mm}$ and $\mathrm{S}=6.9 \mathrm{~mm}$. The mylar thickness is $0.51 \mathrm{~mm}$ and, thus, this figure should be compared to Fig. 9(a). As seen, decreasing the dimensions moved the low frequency dropout up from around $4 \mathrm{GHz}$ to $5 \mathrm{GHz}$. This is an increase by a factor of $1.25(=1 / 0.8)$ which corresponds closely to the change in dimensions D and S. In order to separate the effects of the diameter and the hole spacing, a backshort was made with the diameters increased by a factor 1.1 and the spacing increased by 1.47. The results are shown in Fig. 11(b). The mylar thickness is $1.02 \mathrm{~mm}$ and, thus, this figure should be compared to Fig. 9(e). As can be seen, the deep dropouts have a similar shape but are shifted down in frequency by a factor of $0.92(=1 / 1.09)$ which corresponds to the change in $\mathrm{D}$. This correspondence also held for the two other mylar thicknesses, $0.76 \mathrm{~mm}$ and $0.89 \mathrm{~mm}$, where the upper dropout could be observed. This is consistent with the square hole results that the dropouts are related to the length of the holes. As yet, the theoretical analysis has not been adapted to the case of circular holes.

A few different backshorts with circular holes were tested with both two and three holes. In general, the reflected power in the high reflection band was lower by $0.05 \mathrm{~dB}$ to $0.2 \mathrm{~dB}$ for the two hole case.. This may limit the bandwidth only for the most critical applications. 


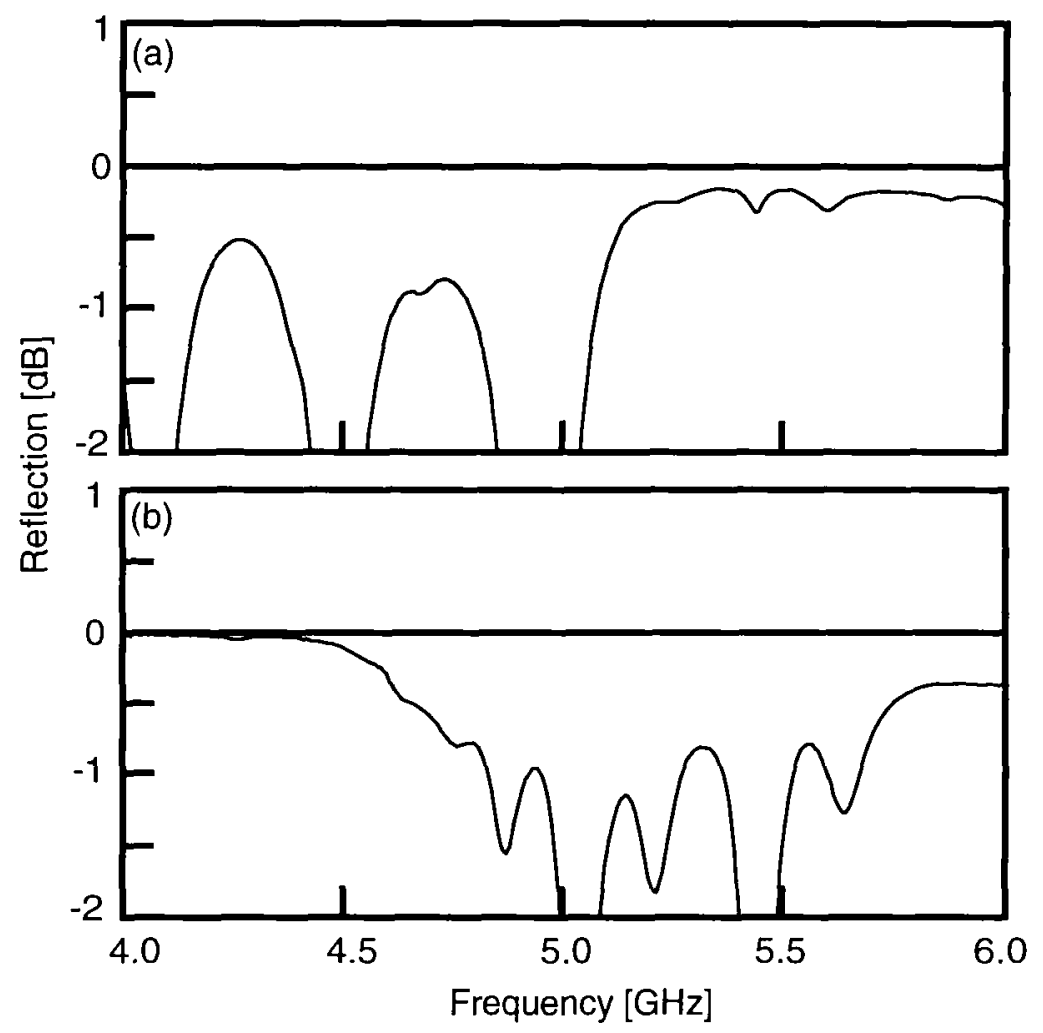

Figure 11. This figure shows the effect of varying the circular hole diameter D and spacing S. In (a), both D and S are decreased by $20 \%$ as compared with the case shown in Fig. 9(a). The mylar thickness is $0.51 \mathrm{~mm}$. In (b), D was increased by a factor of 1.1, and $S$ was increased by a factor 1.47 in order to separate the influence of the hole diameter and spacing. The mylar thickness is $1.02 \mathrm{~mm}$ and, thus, this figure corresponds to Fig. 9(e).

Increasing the number of holes to 4 might give some further improvements but this case was not closely examined. At some point, losses will limit the improvement from adding more holes.

\section{WR-10 Band Measurements}

A crucial test of this new design is to measure its performance at millimeter wave frequencies. The WR-187 band backshorts were scaled for use at WR-10 band using a scale factor of 0.0535. Thus, the backshorts dimensions are $\mathrm{W}=2.51 \mathrm{~mm} x \mathrm{~T}=1.14 \mathrm{~mm}$. The WR-10 
waveguide dimensions are $2.54 \mathrm{~mm} x 1.27 \mathrm{~mm}(0.10$ in. $x \quad 0.05$ in.) and the frequency range $4 \mathrm{GHz}-6 \mathrm{GHz}$ scales up to $75 \mathrm{GHz}-112 \mathrm{GHz}$.

Figure 12(a) shows the reflected power versus frequency for a backshort with 3 rectangular holes with hole dimensions and spacings scaled from the low frequency case. The mylar is $0.051 \mathrm{~mm}$ thick which corresponds to $0.95 \mathrm{~mm}$ at WR-187 band. Thus, the results in Fig. 12(a) should correspond approximately to those shown in Fig. 6(c). As can be seen, the performance is excellent and corresponds well with the low frequency case. The reflection coefficient is $-0.05 \mathrm{~dB}$ to $-0.3 \mathrm{~dB}$ over about a $30 \%$ bandwidth and is suitable for most practical applications. The decrease in reflection near $110 \mathrm{GHz}$ corresponds almost exactly to the

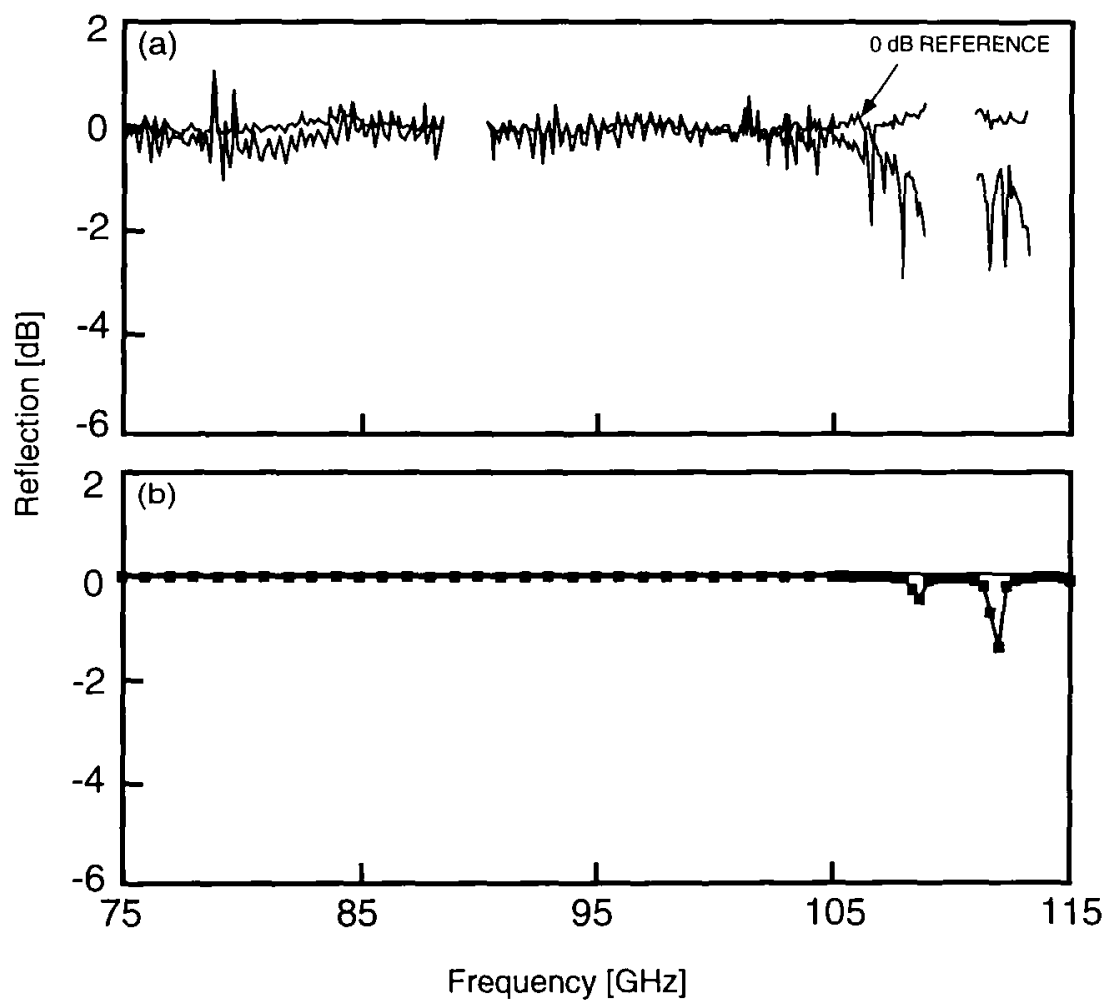

Figure 12. (a) Measured reflected power for a backshort with three rectangular holes in WR-10 waveguide. The mylar is $0.051 \mathrm{~mm}$ thick. The $0 \mathrm{~dB}$ reference is provided by a metal plate inserted between the waveguide flanges. This result corresponds well to the low frequency case, Fig.6(c); (b) Calculated performance of this backshort. The region of high reflection is well characterized by the theory. 
dropout seen near $5.8 \mathrm{GHz}$ in the model. The missing sections of the curves correspond to frequencies at which the BWO was unstable and the data could not be adequately normalized. Also, the small wiggles in the curves are noise and do not result from any resonances in the backshort.

The calculated performance is shown in Fig. 12(b); the theory could not be easily placed directly on Fig. 12(a) since the $0 \mathrm{~dB}$ reference is a measured value which is not a perfectly flat line versus frequency. The theory agrees well with the measurements. As with the low frequency case, the high reflection band is well characterized by the calculations. The predicted dropouts at the high end of the band are smaller than measured, but nonetheless clearly indicate the frequencies at which the performance will begin to degrade.

Figure 13 shows the performance for a backshort with 3 circular holes. These results correspond to the low frequency case shown in Fig. 9 (d). The reflection coefficient is $-0.3 \mathrm{~dB}$ or better over the frequency range from about $76 \mathrm{GHz}$ to $90 \mathrm{GHz}$. Again, this is well suited for many applications. However, this is only about half the bandwidth observed in the low frequency case. The dropout near $105 \mathrm{GHz}$, nonetheless, corresponds well to that seen in Fig. 9(d). The generally low reflection, about $-1 \mathrm{~dB}$, between $90 \mathrm{GHz}$ and $105 \mathrm{GHz}$, is not seen in the low frequency case and may simply result from the mylar thickness not being exactly scaled since only certain thickness are readily available commercially.

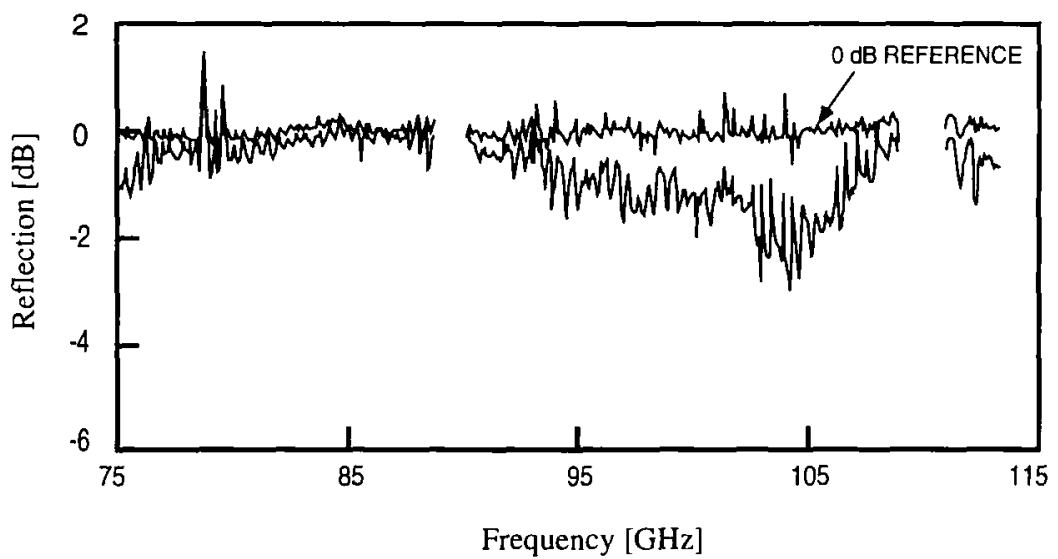

Figure 13. Reflected power for a backshort with three circular holes in WR-10 waveguide. The mylar is $0.051 \mathrm{~mm}$ thick. The $0 \mathrm{~dB}$ reference is provided by a metal plate inserted between the waveguide flanges. This result corresponds well to the low frequency case, Fig.9(d), but the bandwidth is narrower. 


\section{Conclusions}

A novel noncontacting waveguide backshort has been developed which provides excellent performance. It consists of a metallic bar with rectangular or circular holes. It has the advantage of being mechanically rugged and readily fabricated using a variety of methods for frequencies up to the high submillimeter wave range. The dependence of the reflection coefficient and bandwidth on critical backshort parameters has been presented and an optimized design is given which can be scaled to any desired frequency. In general the bandwidth is set by resonances which occur when the electrical length of the holes approaches $180^{\circ}$. The calculated performance using a newly developed theoretical formulation [10] agrees well with the measurements presented here. Thus, either large-scale models or theoretical analysis can be used to design these backshorts to suit a particular application.

\section{Acknowledgements}

We wish to thank K. Jacobs, P. Siegel, and M.A. Frerking for valuable discussions. The research described in this paper was performed by the Center for Space Microelectronics Technology, Jet Propulsion Laboratory, California Institute of Technology, and the NASA Center for Space Terahertz Technology, University of Michigan, and was jointly sponsored by the National Aeronautics and Space Administration, Office of Space Access and Technology, and the Innovative Science and Technology Office of the Ballistic Missile Defense Organization.

\section{References}

[1] M.K. Brewer and A.V. Raisanen, "Dual-harmonic noncontacting millimeter waveguide backshorts: theory, design, and test", IEEE Trans. Microwave Theory Tech. MTT-30, pp. $708-714$ (1982).

[2] A.R. Kerr, "An adjustable short-circuit for millimeter waveguides", National Radio Astronomy Observatory, Electronics Division Internal Report no. 280, pp. 1-25 (1988).

[3] B.N. Ellison, L.T. Little, C.M. Mann, and D.N. Matheson, "Quality and performance of tunable waveguide backshorts", Electronics Letters 27, no. 2, pp. 139-141 (1991).

[4]. Mylar is trademark of the E.I. DuPont Company.

[5]. W.R. McGrath, "A novel noncontacting waveguide backshort for millimeter and submillimeter wave frequencies," Conference Proceedings of the Second National Technology Transfer Conference, NASA Conference Publication 3136, vol. 1, pp. 161 168, December 1991; and W.R. McGrath, K. Jacobs, J. Stern, 
H.G. LeDuc, R.E. Miller, and M.A. Frerking, "Development of a 600 to $700 \mathrm{GHz}$ SIS receiver", Preceedings of the First International Symposium on Space Terahertz Technology, NASA Center for Space Terahertz Technology, University of Michigan, pp. 409 - 433 (1990).

[6]. W.R. McGrath, "Noncontacting Waveguide Backshort," U.S. Patent no. 5138289, 1992.

[7] W.R. McGrath, C. Walker, M. Yap, Y.C. Tai, "Silicon micromachined waveguides for millimeter wave and submillimeter wave frequencies", IEEE Microwave and Guided Wave Letts. 3, pp 61-63 (1993).

[8] T.S. Saad (ed.) Microwave Engineers' Handbook, vol. 1, Artech House, Inc. Dedham, MA, p. 38 (1971).

[9] R.F. Harrington, Time-Harmonic Electromagnetic Fields, McGraw-Hill, New York, pp. 158-161 (1961).

[10]. T.M. Weller, L.P.B. Katehi, W.R. McGrath, "Analysis and design of a novel noncontacting waveguide backshort", to appear in IEEE Trans. Microwave Theory Tech. 